\title{
Dynamic Changes in the Dendritic Geometry of Individual Neurons Visualized Over Periods of Up To Three Months in the Superior Cervical Ganglion of Living Mice
}

\author{
Dale Purves, Robert D. Hadley, and James T. Voyvodic \\ Department of Anatomy and Neurobiology, Washington University School of Medicine, St. Louis, Missouri 63110
}

\begin{abstract}
We describe a means of visualizing the same neuron in the superior cervical ganglion of young adult mice over intervals of up to 3 months. The dendrites of these neurons change during this interval; some branches retract, others elongate, and still others appear to form de novo. Thus, neuronal dendrites in this part of the nervous system are subject to continual change beyond what is usually considered the developmental period. The remodeling of postsynaptic processes further implies that the synaptic connections made onto these cells undergo substantial rearrangement well into adulthood.
\end{abstract}

A great deal of work has indicated that the morphology and synaptic connections of adult vertebrate neurons are capable of considerable change in the face of experimental perturbations. Thus, axon terminals can, in maturity, sprout to form new synaptic contacts in response to a variety of stimuli; alternatively, under other circumstances, terminals can contract their field of innervation (reviewed in Purves and Lichtman, 1985a, Chap. 13). These observations raise the possibility that neuronal connections normally change throughout life. Several lines of evidence support this possibility. First, synaptic rearrangement has been demonstrated in several parts of the mammalian nervous system well into postnatal life (Purves and Lichtman, 1980; Van Essen, 1982). Second, detailed observations of the neuromuscular junction suggest continual changes in the morphology and physiology of these synapses (Anzil et al., 1984; Haimann et al., 1981; Wernig et al., 1980, 1984; see also Townes-Anderson and Raviola, 1978). Third, there are morphological indications of dendritic growth and synaptic remodeling in the adult mammalian brain (see, for example, Sotelo and Palay, 1971).

A major problem in assessing neuronal remodeling in the adult nervous system has been the inability to observe, over time, individual nerve cells and their synaptic connections. In the present work, we describe a method for visualizing the postsynaptic geometry of individual neurons over periods of up to several months in a relatively simple part of the mammalian nervous system, the superior cervical ganglion. This approach involves (1) identification of an individual nerve cell in the living ganglion at intervals of days to months; (2) visualization of the dendritic geometry of the cell by injection of a nontoxic fluorescent dye; and 3) rapid acquisition and analysis of images of

\footnotetext{
Received July 1, 1985; revised Sept. 9, 1985; accepted Sept. 10, 1985.

This work was supported by USPHS Grants 11699 and 18629 and a grant from the Muscular Dystrophy Association. R.D.H. was supported by NIH Training Grant F32-NS07297; J.T.V. is supported by an NSF Graduate Fellowship. We are grateful to C. Forehand, W. Harris, J. Lichtman, J. Sanes, W. Snider, and H. Yawo for useful comments, and to C. Forehand for supplying the electron micrograph shown in Figure 1. Pat Newton provided valuable technical assistance.

Correspondence should be addressed to Dale Purves, Department of Anatomy and Neurobiology, Washington University School of Medicine, 660 South Euclid Avenue, St. Louis, MO 63110.
}

Copyright (C) 1986 Society for Neuroscience $0270-6474 / 86 / 041051-10 \$ 02.00 / 0$ the dendritic arbor to enable comparison of the cell's configuration at two different times.

Our results in a large number of sympathetic ganglion cells visualized in living mice indicate that the dendrites of these cells gradually change in young adult animals. These changes involve the extersion of some branches, the retraction and disappearance of others, and the de novo formation of still other dendrites. Since preganglionic synapses are made primarily on dendrites in the sympathetic ganglia of mice and other mammals (Forehand, 1985), these ongoing changes suggest a commensurate change in the distribution of synaptic endings.

Some of these results have been briefly reported (Hadley and Purves, 1985; Purves and Hadley, 1985).

\section{Materials and Methods}

Identification of individual superior cervical ganglion cells in the living mouse

Autonomic ganglia provide an especially favorable site for visualizing individual nerve cells in life because many neuronal cell bodies lie superficially, covered only by a thin capsule (Fig. 1). In order to see these cells in situ, young adult male mice (CF1 strain, 25-35 gm, 8-15 weeks old) were anesthetized with chloral hydrate $(0.6 \mathrm{gm} / \mathrm{kg})$. This dose provides deep surgical anesthesia within 2-3 min. The neck was shaved and the animal mounted on a stage that could be moved in three axes by a micrometer drive (Fig. $2 A$ ). The trachea was intubated with a fine polyethylene tube introduced through the mouth and the animal ventilated by positive pressure throughout the procedure. The skin was surgically prepared and a midline incision made from the sternal notch to the apex of the mandible. The superior cervical ganglion was exposed and gently retracted so as to place it in the center of a pool of saline that was grounded with a silver-silver chloride paddle (Fig. $2 B$ ). Individual cells on the ganglionic surface were visualized by placing a $\mathrm{fi}$ beroptic light guide $(0.7 \mathrm{~mm}$ diameter $)$ just beneath the medial edge of the ganglion (Fig. 2, $B$ and $C$ ). The tip of the light guide was beveled and polished at a $45^{\circ}$ angle and the end coated with reflecting white enamel. As a result, the light from the tip of the pipe emerged at an angle tangential to the ganglionic surface, providing a high-contrast image by asymmetric illumination contrast (see, for example, Kachar, 1985). By adjustment of the position of the light, individual cells across most of the ganglionic surface could be clearly seen (Fig. 3 ).

The mouse superior cervical ganglion comprises about 10,000 neurons (Purves et al., 1986); therefore, the selection of any particular cell for study was arbitrary. However, we usually chose a relatively large neuron located at the surface of the ganglion that, by virtue of its position and relationship to blood vessels, would be easy to identify when we searched for it later (see Fig. 3). Having selected a cell, we took $35 \mathrm{~mm}$ color transparencies of the ganglionic surface at 5,125, and $250 \times$; the respirator was turned off for a few seconds during photography to minimize movement (there was still a slight movement arising from the pulse even though it was damped by gentle retraction-see Fig. 2). The low-power photomicrographs were useful in locating the proper region of the ganglion at a second operation. The higher-power photos, together 


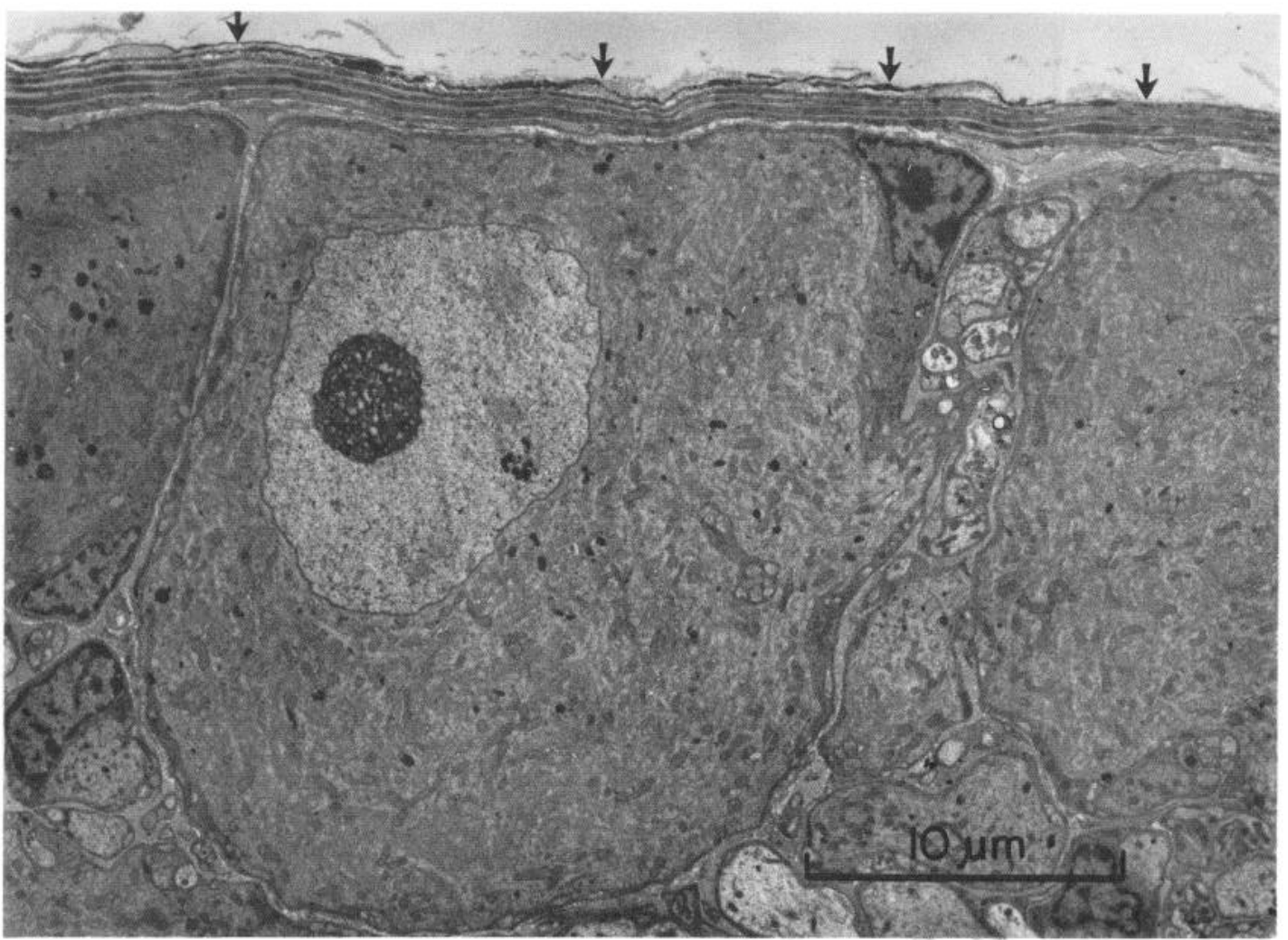

Figure 1. Electron micrograph of the surface of an adult mouse superior cervical ganglion (cross section). Once the ganglion has been exposed in situ (see Fig. 2), only a thin capsule (arrows) lies between the observer and the neurons of interest. Surface neurons such as the ones shown here are visualized in vivo by asymmetric illumination contrast (see Fig. 3).

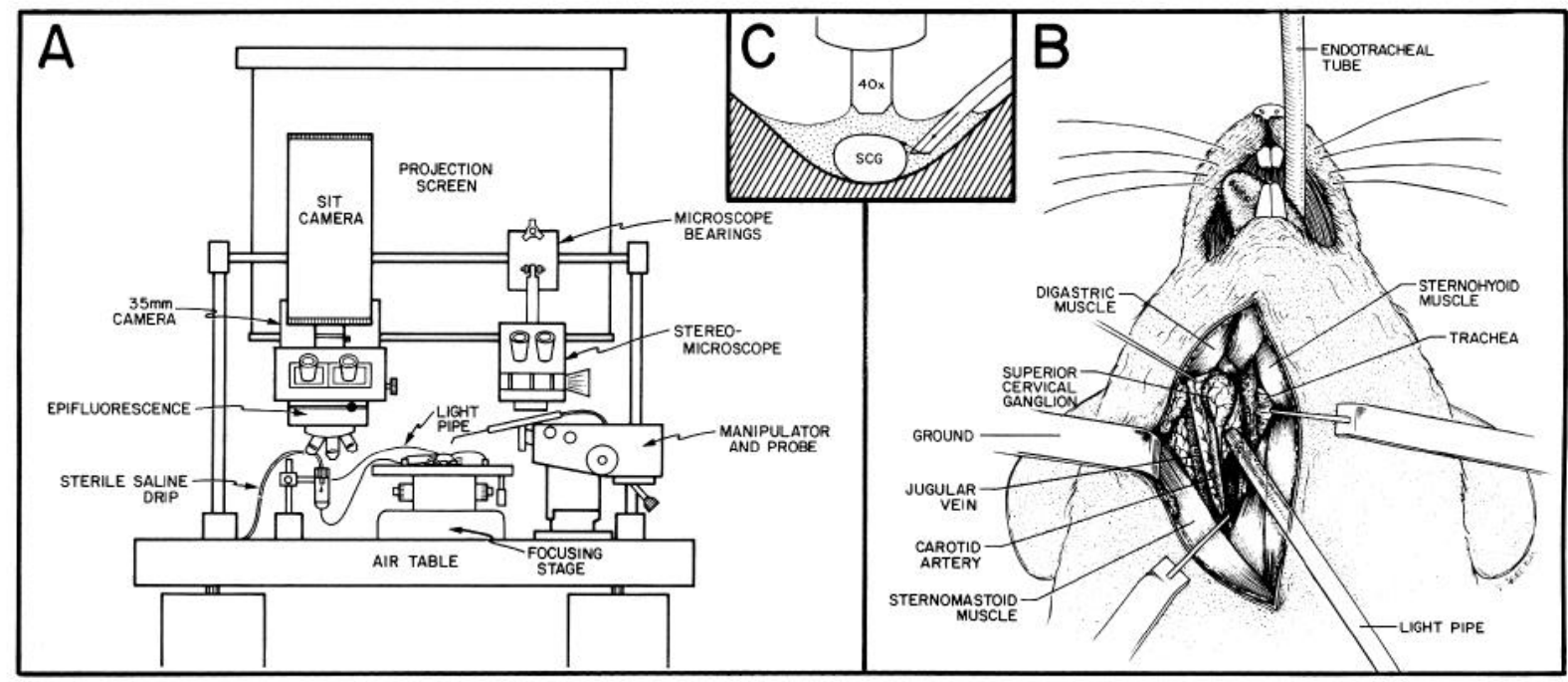

Figure 2. Technical approach to viewing individual neurons in the superior cervical ganglion of the living mouse. A, Apparatus used for in situ viewing of individual neurons. A stereomicroscope and a compound microscope can be quickly and smoothly interchanged by means of a track and roller bearings. Dissection and electrode placement are carried out under the dissecting microscope; the compound microscope, fitted with a low-light-level video (SIT) camera as well as a $35 \mathrm{~mm}$ camera, is used for neuronal impalement and subsequent viewing. This arrangement allows permanent recording of the surface position of the neuron chosen for study and video monitoring and photography of the cell when it has been filled with dye. In order to reidentify the cell at a later time, the photomicrographs of the ganglion surface taken at the first operation are projected onto a screen in front of the setup, while the experimenter views the ganglion through the compound microscope at a second operation. $B$, The mouse is placed on a focusing stage (see $A$ ) (from a disassembled compound microscope) and the superior cervical ganglion exposed as shown. The carotid artery is gently retracted with a blunt hook to minimize the transmitted pulsation; the ganglion is superfused with sterile saline (see $A$ ) throughout the procedure. $C$, Arrangement of the light pipe necessary to achieve asymmetric illumination contrast images of superior cervical ganglion cells (see Fig. 3). By careful adjustment of the light, an optimal position for visualizing particular surface cells with high contrast can be determined; arrows indicate the direction of illumination. The tip of the light pipe is placed just under the medial edge of the ganglion (SCG), as illustrated. 

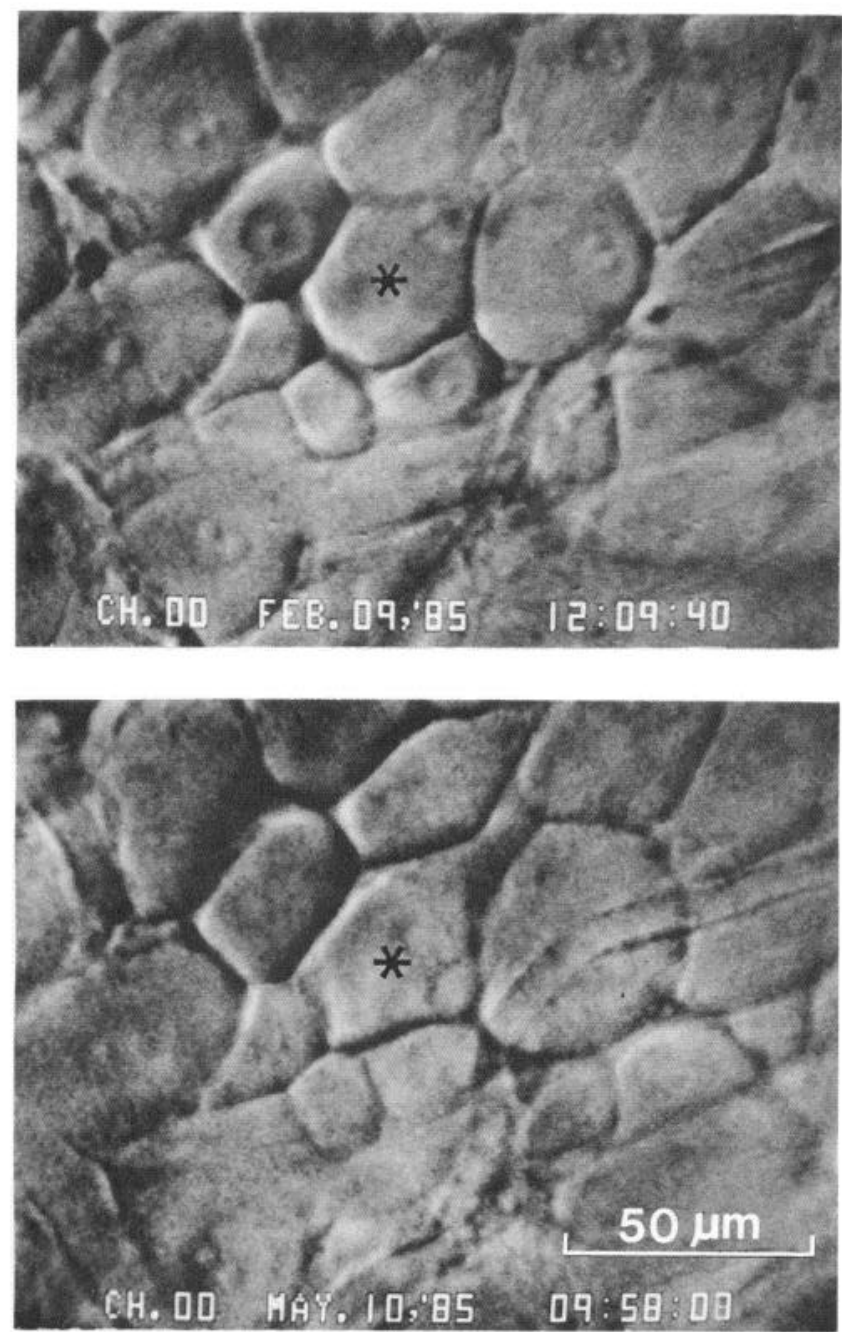

Figure 3. Surface images of the living superior cervical ganglion visualized on a video monitor with the apparatus shown in Figure 2. Examples are from the surface of a ganglion observed over an interval of 3 months (images have been enhanced as described in Materials and Methods). Neurons appear as a mosaic in which individual cells can be recognized by their shape, neighbors, and relation to nearby landmarks, such as blood vessels (cf. Fig. 1). The cell chosen for study in this particular ganglion is indicated by the asterisk; the size, shape, and neighbor relationships of individual cells usually make reidentification unambiguous.

with a high-resolution surface image obtained with a video camera, allowed us to re-identify the cell of interest without ambiguity (Fig. 3; see also below)

\section{Intracellular injection of fluorescent dye and visualization of} dendritic arbors with low-light video microscopy

Once we had obtained photographs of the ganglionic surface, a microelectrode was carefully positioned over the center of the cell of interest (see Fig. 2A). Electrodes pulled from triangular glass (Glass Company of America) were filled with a 5\% solution of 5(6)-carboxyfluorescein in $0.44 \mathrm{M}$ potassium hydroxide ( $\mathrm{pH} 7.0$ ) that had been passed through a Millipore filter $(0.2 \mu \mathrm{m})$. These electrodes had resistances of $80-140$ $\mathrm{M} \Omega$. The neuron was impaled by passing a brief pulse of hyperpolarizing current while pressing lightly on the cell surface. Virtually every cell impaled in this manner had a resting potential of -40 to $-60 \mathrm{mV}$ and showed overshooting action potentials whose amplitudes were at least $60 \mathrm{mV}$ (Fig. 4).

Carboxyfluorescein was iontophoretically injected by a direct current of $3 \mathrm{nA}$ delivered for a period of $2 \mathrm{~min}$; the majority of cells maintained

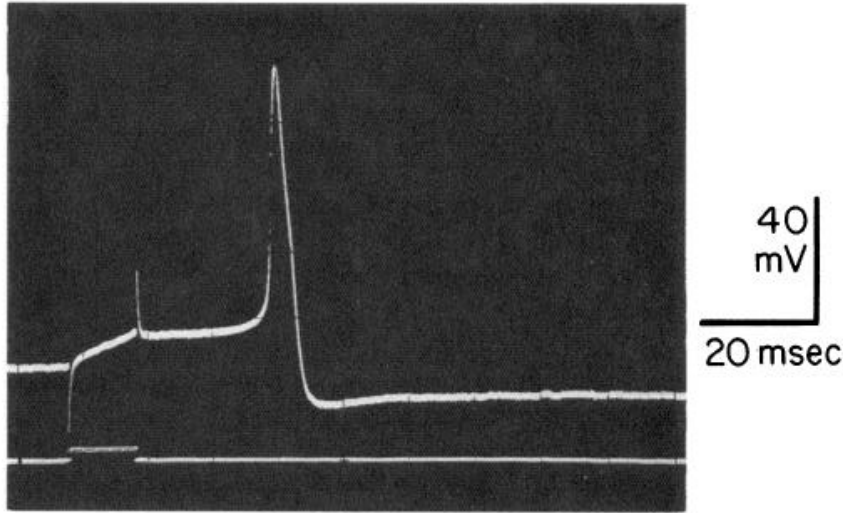

Figure 4. Intracellular recording from a mouse superior cervical ganglion cell in situ. Under the circumstances described in Figure 2, stable recordings could be obtained routinely. Neurons showed no spontaneous activity or synaptic potentials; this quiescence was presumably a consequence of the depth of anesthesia that was maintained. Action potentials of normal amplitude occurred for some seconds after impalement and afterward could be elicited by depolarizing current injection, as shown here. Resting potential, $-52 \mathrm{mV}$.

their resting potential during this time. The dye is not retained for very long; the fluorescent intensity of labeled cells faded appreciably within a few hours of injection, and was no longer apparent after 12-24 hr.

Further illumination was delivered through a $530 \mathrm{~nm}$ long-pass filter to remove wavelengths that would excite the fluorescent dye. This enabled visualization of the ganglion surface without inducing photooxidative damage in the labeled neuron. A compound microscope with a $40 \times$ water-immersion objective (Zeiss, 0.75 numerical aperture, 1.6 $\mathrm{mm}$ working distance) was then placed over the ganglion and focused on the injected cell. A final, higher-resolution surface picture of the injected cell and its neighbors was made using a low-light video camera (GE SIT camera, model 4TE56; see Fig. 3). By briefly illuminating the field with epifluorescence optics, we could confirm that the intended cell had, in fact, been filled.

For epifluorescence microscopy, the light from a $15 \mathrm{~W}$ tungsten bulb was passed through two heat filters and conventional exciter and barrier filters for fluoresceinated dyes (Leitz H2 cube; 390-490 nm excitation; $515 \mathrm{~nm}$ suppression). The purpose of the low-wattage illumination was again to minimize photo damage of the injected neuron (Kater and Hadley, 1982a, b). Using the low-light video camera and viewing the injected cell on a video monitor, we then selected a region of the dendritic arbor that was near the surface and easily seen. This usually involved one primary dendrite and its higher-order branches, although occasionally we studied the entire dendritic arborization of a cell. In general, the length of the dendritic branches studied was about half of the average total dendritic length of mouse superior cervical ganglion cells (Purves and Lichtman, 1985b).

A series of optical sections through the branches of interest was obtained using an image processor and a computer (Fig. 5; see also below). Ten to fifteen such sections were acquired within a minute or two, resulting in a total exposure to the exciting illumination of about 30 $\mathrm{sec}$. These observations were usually made about $10 \mathrm{~min}$ after dye injection. Filling of neuronal processes by diffusion appeared to be complete at even shorter intervals; thus the arbors seen with fluorescent optics a few minutes after dye injection were similar to those observed when mouse superior cervical ganglion cells were filled with HRP and visualized in fixed and cleared ganglia (Purves and Lichtman, 1985b).

Once the images had been stored, the microscope was removed and the wound closed with fine sutures. The operating time, from anesthesia to wound closure, was on the order of an hour. The animal was then warmed with a heat lamp and the endotracheal tube removed; most animals awoke about a half-hour later.

\section{Relocation of the injected neuron}

After an interval of 3-90 d, the animals were again anesthetized and the procedure repeated exactly. The photomicrographs of the ganglion surface taken at the original operation were projected onto a screen that 

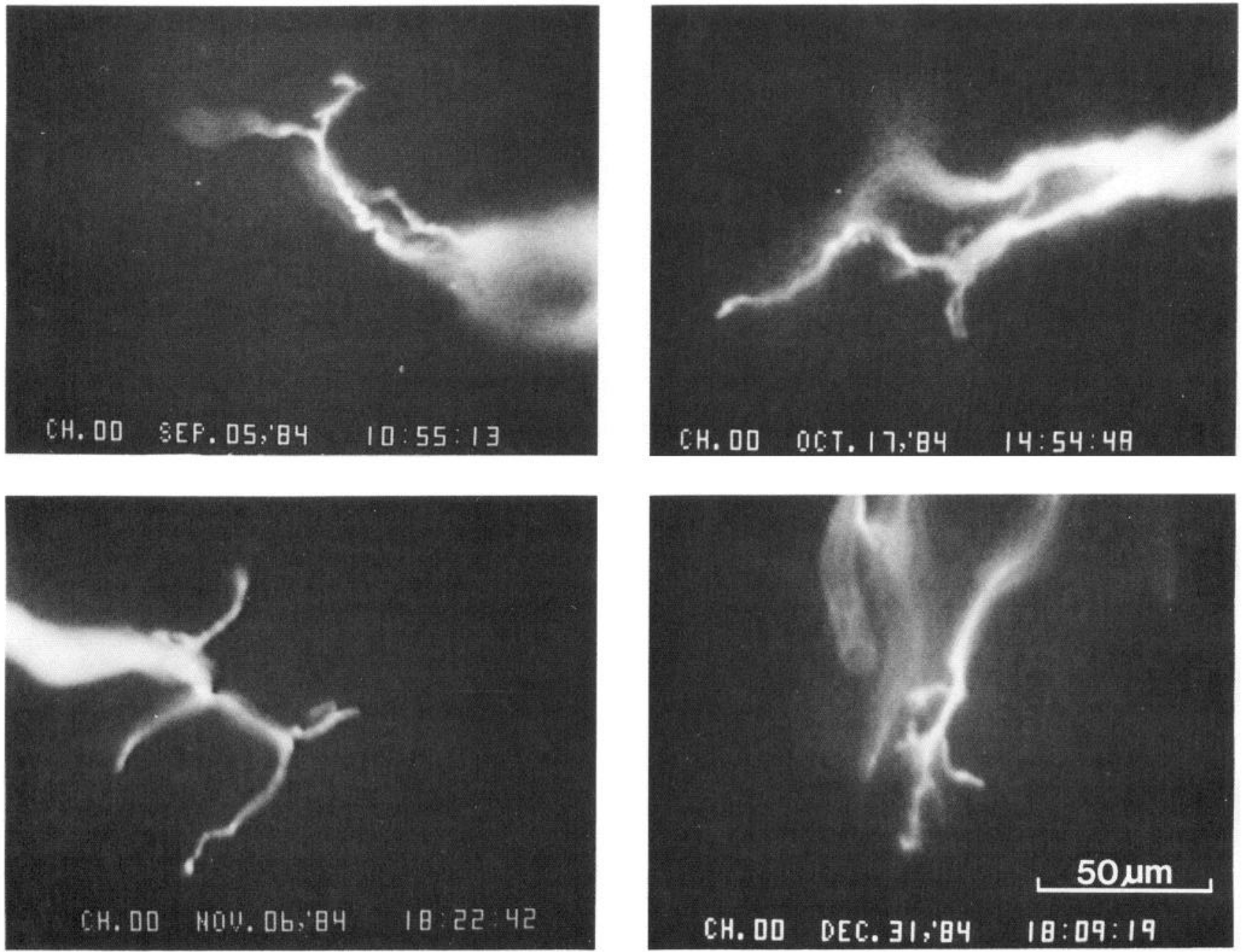

Figure 5. Low-light-level photomicrographs taken from the video monitor show single optical sections through a portion of the dendritic arbor of four different superior cervical ganglion cells. The pictures were taken about $10 \mathrm{~min}$ after injection of the neuronal cell body with 5(6)carboxyfluorescein. These examples were chosen to show that the terminal portions of the dendritic arbors appear to be filled completely; delicate terminal branches on the order of $1 \mu \mathrm{m}$ in diameter are easily seen. The dendritic arbors observed in this way were not different in appearance from mouse superior cervical ganglion cells visualized in vitro after filling with HRP (Purves and Lichtman, 1985b).

faced the electrophysiological setup (Fig. $2 A$ ); by comparing the topography in the photographs with the image under the microscope, we could usually find the cell that had been injected originally within a few minutes. Once the neuron of interest had been located (see Fig. 3), it was impaled and injected as at the initial operation. The range of resting potentials and action potential amplitudes recorded in the great majority of re-impaled cells was not appreciably different from the values obtained at the initial operation (resting potentials, -40 to $-60 \mathrm{mV}$; action potentials, $60-120 \mathrm{mV}$ ). The portion of the dendritic arbor imaged previously was then located and fluorescent pictures obtained as before. In all our experiments the animal was killed at the end of this procedure.

\section{Image analysis and morphometry}

Video images of the dendrites, as well as those of the ganglion surface (see above), were collected and manipulated using a Grinnell image processor (model 274) attached to a PDP 11/44 computer (Digital Equipment Corp.). A general-purpose image-analysis language (IMAGR; J. Voyvodic, unpublished) controlled the image processor. This system provided several important advantages: (1) video noise was greatly reduced by signal-averaging each image $32 \times$ during digitization, (2) images could be acquired and stored in computer disk files very rapidly ( $4 \mathrm{sec} / \mathrm{image}$ ), (3) video image quality could be enhanced by contrast expansion, and (4) for images of the ganglionic surface, asymmetries in the overall illumination could be removed by digitally subtracting an out-of-focus background image from the in-focus surface image (see Fig. 3).
Comparisons of the geometry of the neuron of interest over the interval chosen were made by reconstructing the relevant portion of the dendritic arbor from the optical sections obtained at each of the two operations. For this analysis, the stored images of the dendrite were recalled, viewed on the monitor, and photographed on Polaroid film (see Fig. 5). The pictures were projected onto a piece of paper using an artist's projector (Artograph RT 200); in this fashion we could make detailed two-dimensional drawings of the cell we had studied. Morphometric analysis from reconstructions of the dendrites was performed using the image-analysis language with a digitizing tablet attached to the computer.

\section{Results}

Survival of neurons filled with fluorescent dye and visualized in situ

A single neuron in the superior cervical ganglion was injected and visualized in each of 165 young adult mice. In 148 of these animals we were successful at the second operation in identifying the region of the ganglionic surface that had been studied previously. In 17 animals we were unable to locate the appropriate area and therefore could not determine whether the cell initially injected had survived. Most of these failures occurred before we became experienced at choosing locations and cell groupings that we could easily re-identify. In 91 of the 148 animals in 


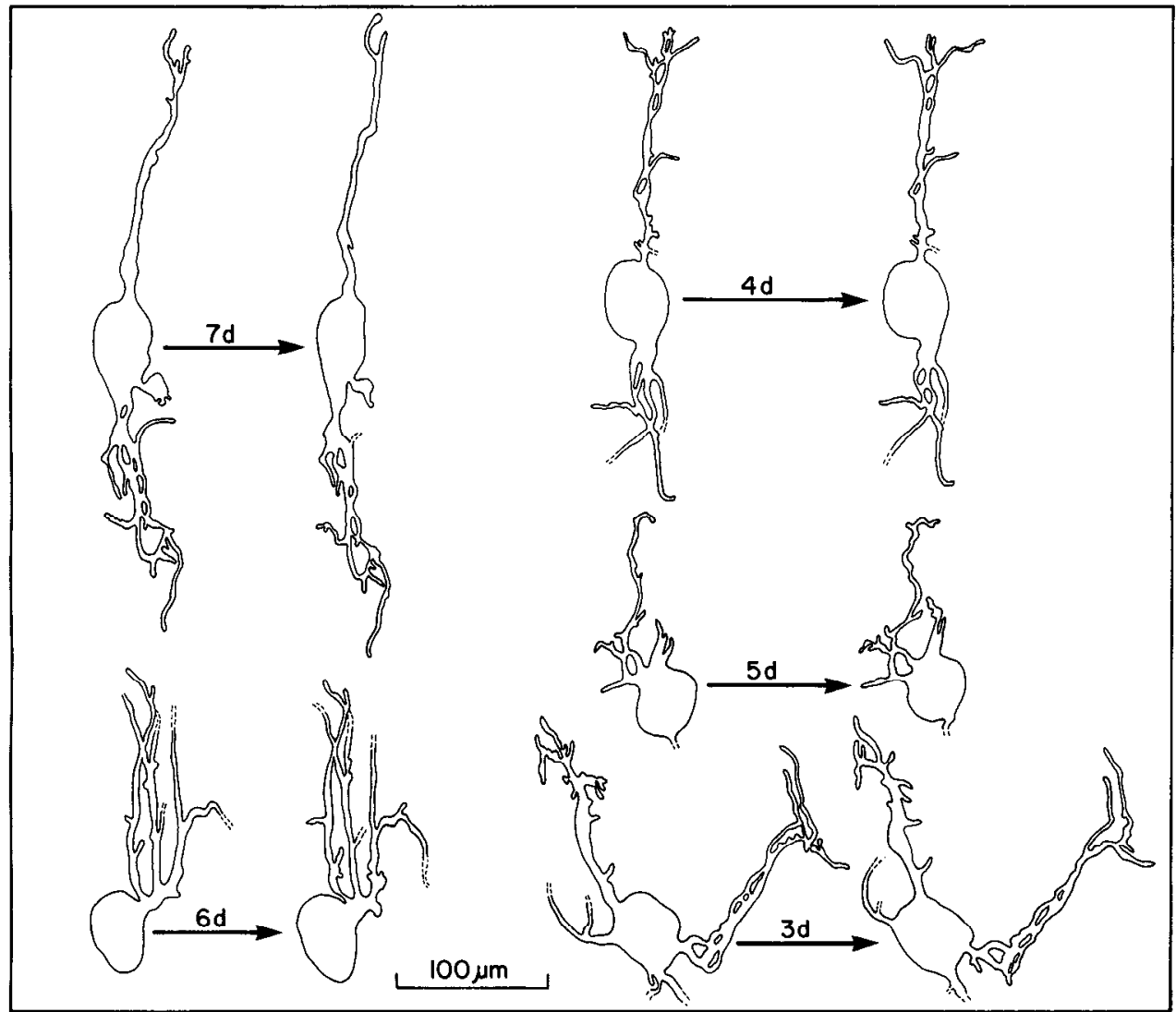

Figure 6. Reconstructions of selected portions of the dendritic arbor of five representative neurons visualized after intervals of a few days. In this and subsequent figures, 5-15 optical sections, such as those shown in Figure 5, were combined to make two-dimensional projections of the dendritic arbor of the cell of interest. No attempt was made in these reconstructions to indicate variations in the third dimension; dashed lines in this and subsequent figures indicate processes that continue but were not chosen for study. Over intervals of a few days, the dendritic arbors change very little. which the correct region was located, the cell of interest was identified and successfully re-impaled with a dye-filled microelectrode. In the remaining 57 cases, the neighboring cells could be identified but the cell of interest had apparently died and been removed by phagocytic action. Thus, the survival rate of neurons studied in this way was $61 \%$. The disappearance of a cell of interest occurred with about the same frequency at the shortest intervals examined (a few days) as at longer intervals (up to 3 months); evidently most cells that die as a result of impalement and/or illumination do so promptly.

Prolonged or intense exposure of the fluorophore to the exciting wavelengths can certainly kill a dye-filled cell (see, for example, Miller and Selverston, 1979). However, the death of about $39 \%$ of the neurons initially impaled is probably not explained simply by illumination of the fluorescent dye, since in additional control experiments 4 out of 10 cells that were filled with dye but never illuminated had also degenerated and been entirely removed when these ganglia were examined 2 weeks later. Although we cannnot exclude the possibility that the dye alone has some toxicity, 5(6)-carboxyfluorescein is apparently nontoxic in other neural systems (Kater and Hadley, 1982a, b; McPheeters and Okun, 1980; Okun, 1981). In 10 other animals, a neuron was impaled with a microelectrode containing only potassium chloride $(0.5 \mathrm{M})$. Again, 2 out of 10 of these cells could not be found 2 weeks later, implying that impalement alone is, in some instances, sufficient to precipitate neuronal degeneration and phagocytic removal. In animals in which the surface of the ganglion was exposed and photographed, but the cells not impaled, only 2 out of 131 neurons had disappeared when the ganglionic surface was visualized again several weeks later. Whether the loss of occasional cells in these sham experiments represents a normal process or inadvertent damage is not known.

Finally, 8 of the 91 cells examined showed some evidence of injury upon reimpalement (shrinkage of the cell soma, leakage of dye, or resting potential less than $-40 \mathrm{mV}$ ). Seven of these cases were cells reexamined within a few days; however, one such cell was observed after an interval of $18 \mathrm{~d}$. Some of these instances of apparent damage may simply have been the result of poor technique at the second operation (the ganglionic surface is somewhat more difficult to see clearly when the wound is still healing). However, a conservative explanation would be that the effects of damage after an initial impalement can sometimes persist for a number of days. In any event, the eight cells judged to show evidence of injury at re-impalement were not considered further. The related question of whether sublethal damage affects the results we describe below is considered in the Discussion.

\section{Changes in neurons visualized at} intervals of 3-10d

Twenty-four neurons were observed after intervals of only a few days to assess whether changes in the configuration of dendrites occur over relatively brief times. Most of the branches seen initially could be identified after a few days and were approximately the same length and shape (Fig. 6; see also Fig. 14 below). In each cell, however, small differences were apparent. Given the fact that at any two viewings the ganglion is never in exactly the same orientation or under exactly the same tension from the retractors (see Fig. $2 B$ ), it remains uncertain whether these small discrepancies seen at early times are simply due to differences in rotation or tension on the dendritic tree, or whether they represent real changes in dendritic configuration.

Neurons visualized at intervals of 2-3 weeks

The 20 cells that were visualized at an interval of 13-18 d were also easily recognized as having the same overall configuration as initially. However, examination of these cells often revealed differences in dendritic geometry that exceeded changes easily 

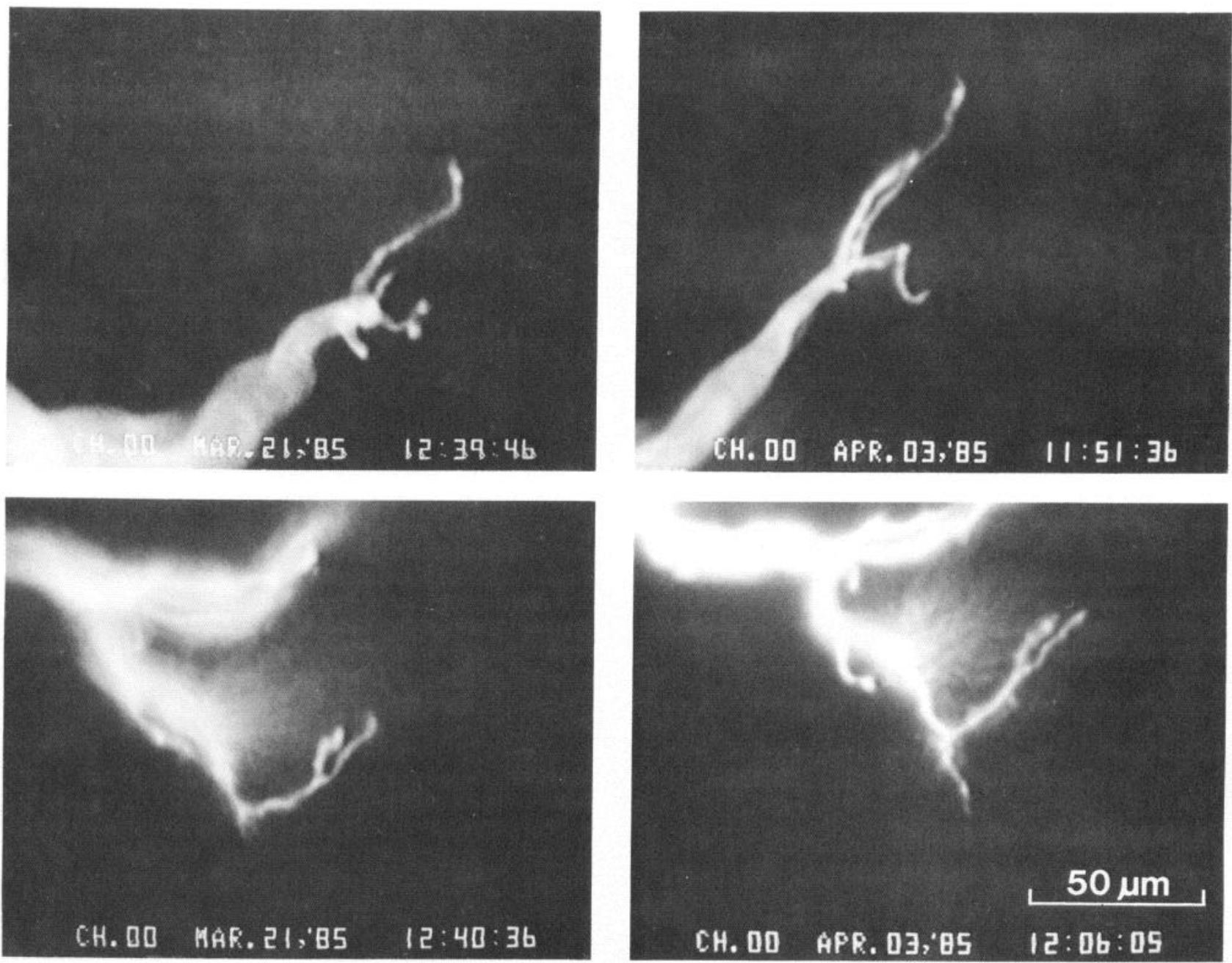

Figure 7. Comparable focal planes of two different portions of the dendritic arbor of a cell visualized at an interval of about 2 weeks. Changes in shape that would be difficult to explain by differences in rotation or tension are now apparent.

attributable to differences in orientation or tension on cell processes (Figs. 7 and 8). The changes observed at this interval involved the retraction of some branches and the extension of others. In some instances, small branches evident during the initial observation of the cell had disappeared altogether, whereas other dendritic branches appeared de novo in the 2 week interval (Fig. 8; see also Fig. 2 in Purves and Hadley, 1985).

\section{Neurons visualized at intervals of 1-3 months}

Cells were imaged at intervals of 29-33 d in 18 animals (Fig. 9), at 57-63 d in 12 animals (Fig. 10), and at 87-90 d in 7 animals (Fig. 11). Although the general configuration of dendrites at the second viewing could still be recognized as similar to the initial pattern, in most cases the changes were more extensive than those observed at shorter intervals (cf. Figs. 6-11). In general, the changes appeared to be progressive over the entire interval, with more extensive differences at 2 months than at 1 month, and still more at 3 months than at 2 (see next section). The changes observed at these intervals again involved a combination of extension, retraction, and de novo formation of branches.

\section{Quantification of dendritic change over} intervals of up to 3 months

Our results suggest that changes in neuronal dendrites are ongoing in young adult life, and that these alterations involve both extension and retraction of neurites. In order to quantify these changes, we measured several aspects of the reconstructed images, examples of which are shown in Figures 6 and 8-11. Figure 12 shows the changes in overall length of the dendritic branches as a function of the interval between observations. With time, the dendritic length deviates increasingly from the original determination; there is, on average, a net increase in length of $26 \%$ at intervals of 2-3 months. As a measure of dendritic complexity, the number of terminal dendritic branches was also determined. The increase in complexity with longer intervals (Fig. 13) implies that much of the change in dendritic length can be acounted for by increased branching within the arbor (cf. Figs. 12 and 13).

It was more difficult to quantify the degree to which individual branches had retracted, extended, disappeared, or formed de novo, since such measurements are predicated on the identification of corresponding elements in the images obtained at an initial and a second viewing. At the longer intervals, the identification of corresponding branches could not always be made with confidence because the changes observed were often substantial (see Figs. 9-11). Accordingly, we selected those cells in which identity between one or more branches over the interval was obvious. We then measured the changes in length for each of these homologous segments. Figure 14 indicates that progressive retraction and extension among homologous branches are both common, although, on average, extension is more fre- 

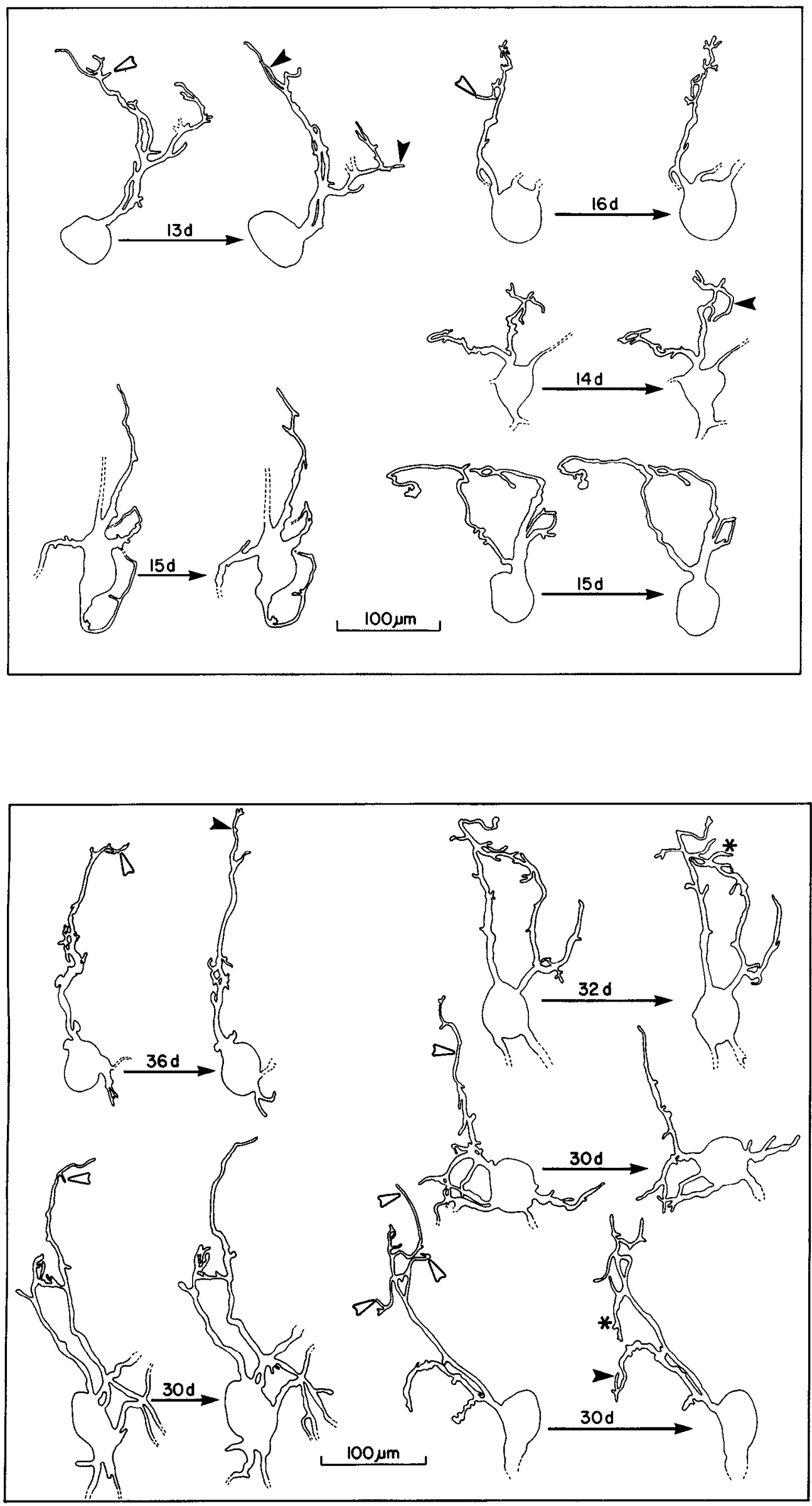

Figure 8. Reconstructions of selected portions of the dendritic arbors of five representative neurons visualized over an interval of about 2 weeks. Changes involving both the extension and retraction of particular branches are evident at this interval. Open arrowheads (here and in Figs. 9-11), examples of a branch that appears to have retracted; closed arrowheads, some examples of branches that appear to have extended.
Figure 9. Reconstructions of selected portions of the dendritic arbors of five representative neurons visualized over an interval of about 1 month. The changes observed were typically greater than those seen at shorter intervals (cf. Figs. 6-8). Symbols as in Figure 8; an asterisk indicates a branch that has evidently formed de novo. 
Figure 10. Reconstructions of selected portions of the dendritic arbors of five representative neurons visualized over an interval of about 2 months. Symbols as in Figure 9.

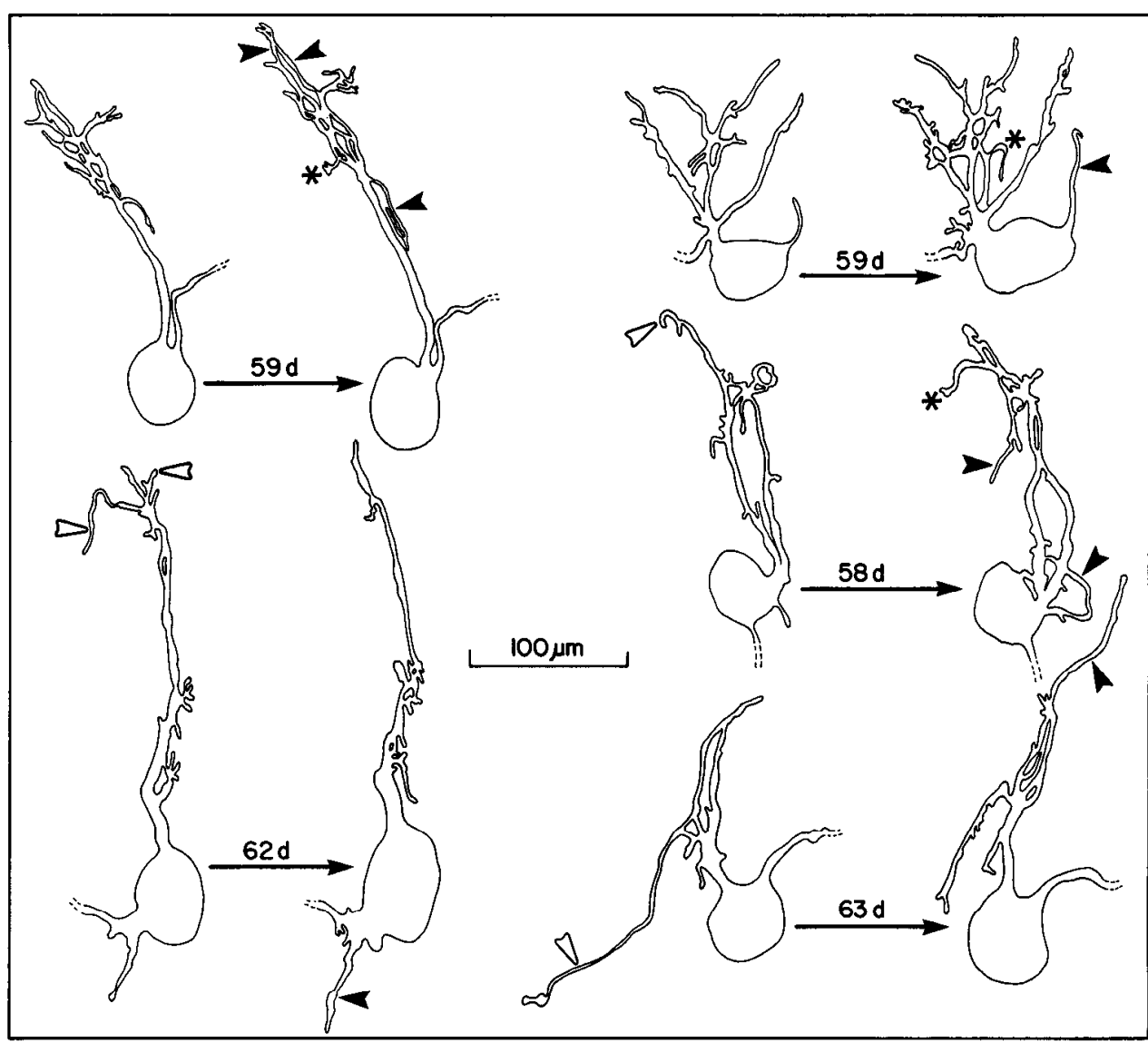

Figure 11. Reconstructions of selected portions of the dendritic arbors of five representative neurons visualized over an interval of about 3 months. The changes are more obvious, and an overall tendency toward greater length and complexity is now apparent. Symbols as in Figure 9.

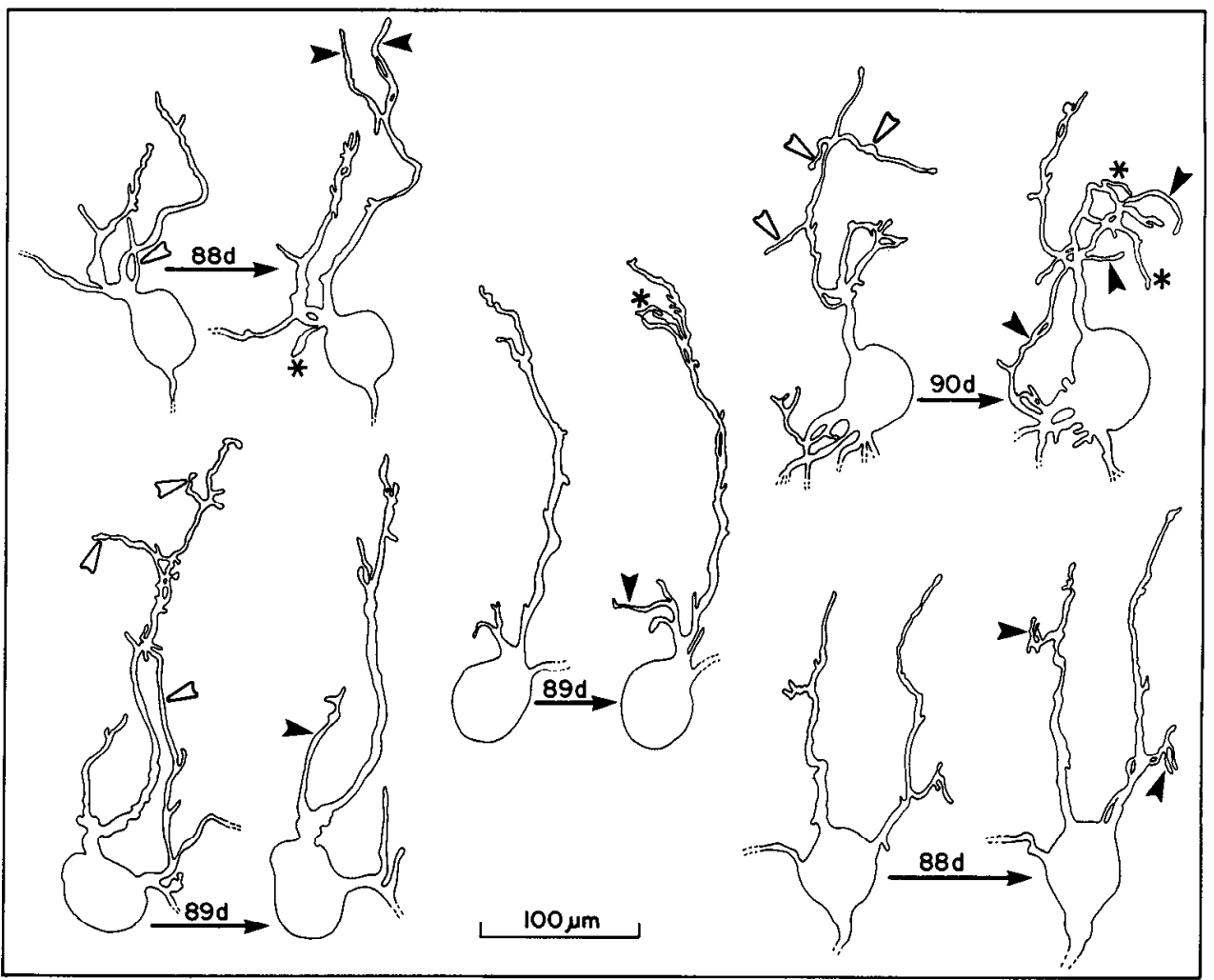




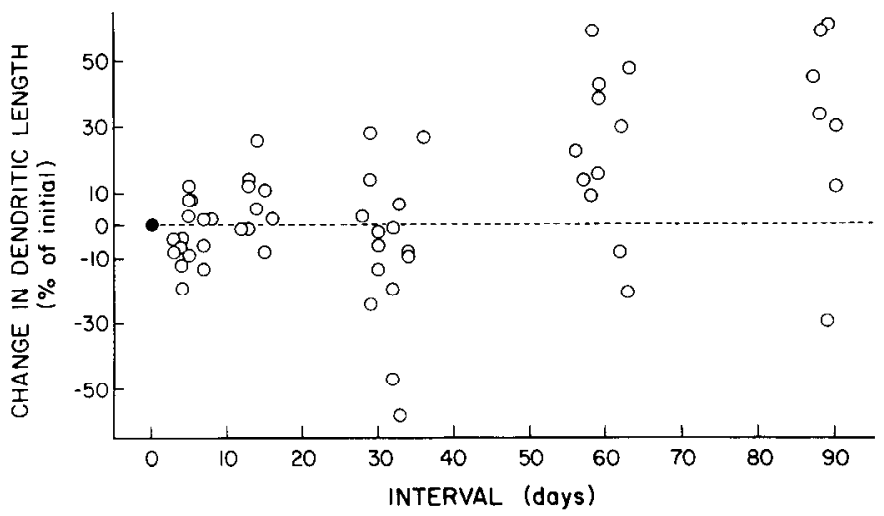

Figure 12. Quantitative summary of the change over time of the overall length of the dendritic arbors of individual ganglion cells visualized in vivo. Each open circle represents a different neuron; the filled circle at the zero position of both axes represents the initial lengths. As the interval between observations becomes longer, the change in length deviates progressively from the initial length. For an individual cell, the net change can be positive or negative; on average, however, a net increase in length is apparent at longer intervals.

quent. These changes of individual branches are consistent with the net increase in the total length of dendritic arbors observed over time (see Fig. 12). The changes shown in Figure 14 underestimate the average change in particular branches, since the elements most easily recognized as homologous over a given interval were necessarily those instances where the change was least.

\section{Discussion}

The results we describe complement a number of less direct observations which suggest that ongoing changes in neuronal geometry and synaptic connections are commonplace in the vertebrate nervous system (see the introduction). In the following discussion, we first consider some technical issues that bear on the validity of our conclusion that the dendrites of superior cervical ganglion cells in the mouse normally change over periods measured in weeks and months. We then consider the more general implications of such changes in the nervous system.

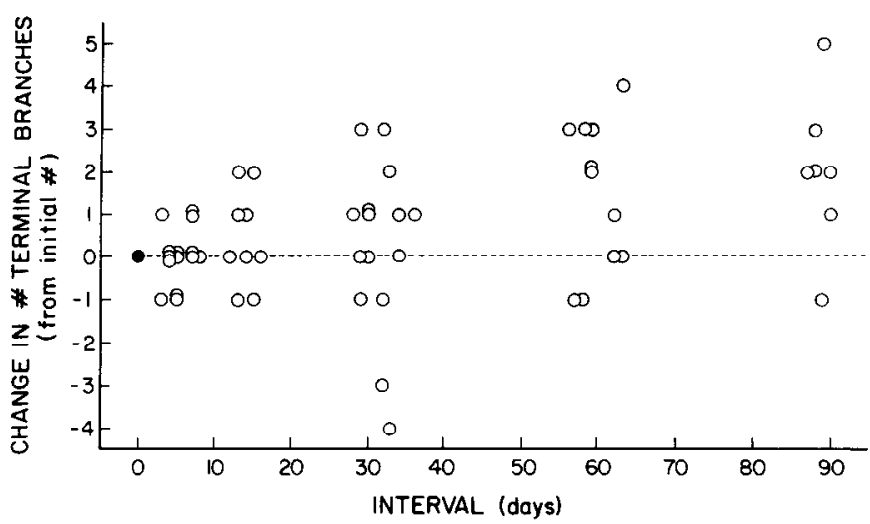

Figure 13. Quantitative summary of the change over time in the number of terminal branches occurring in the dendritic arbors of individual ganglion cells visualized in vivo (same neurons as in Fig. 12). A terminal branch was defined as a process more than $10 \mu \mathrm{m}$ in length with a free ending; the filled circle at the zero point of both axes represents the initial number of branches. By this measure, the average complexity of these cells tends to increase at longer intervals.

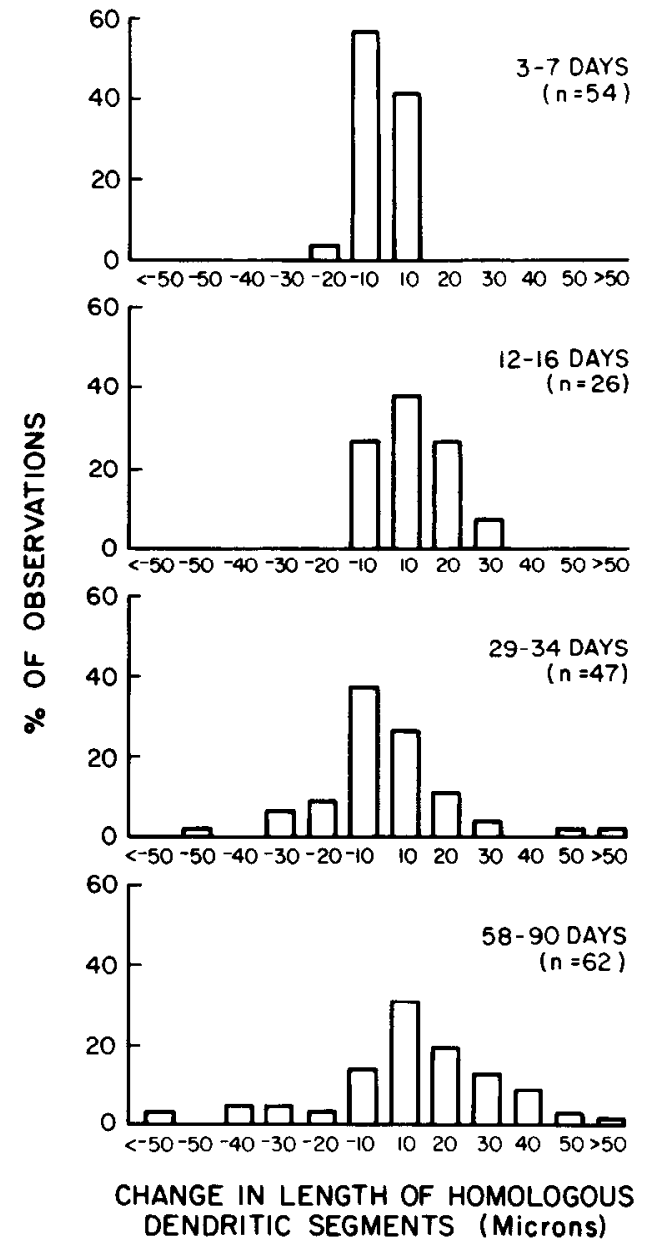

Figure 14. Changes in the length of homologous dendritic segments over time. Only dendritic segments (both terminal and nonterminal) that could be unambiguously identified as homologs were considered. Therefore, the number of cells and branches included here is much smaller than the total sample. The length of particular segments tends to change progressively; for the reasons stated in the text, the estimated degree of change is conservative.

\section{Ongoing dendritic changes are probably not an artifact arising from our procedure}

A central question is whether the changes in neuronal geometry that we describe reflect the normal behavior of these cells. Obviously, the procedure we have used can cause substantial damage to the impaled neurons; indeed, $39 \%$ of the neurons studied in this way promptly succumbed and were removed, while occasional cells showed some signs of injury for several days after microelectrode impalement and visualization. Might the changes we observed be entircly explaincd as a response to ccll damagc?

Several aspects of our results argue against this interpretation. First, and most important, the dendritic arbors showed few differences initially, but changed slowly and progressively over periods of up to 3 months. Although our procedure probably caused some damage to even the surviving cells, it secms unlikely that injury associated with impalement and visualization would cause continued and progressive changes in the configuration of neuronal dendrites over periods of several months. Second, the majority of the cells that survived the procedure appeared to remain healthy: the resting potentials and action potential amplitudes recorded in most identified neurons at the second operation were indistinguishable, on average, from values obtained during the initial impalement. Third, the dendritic arbors of surviving cells, although changed at longer intervals, 
were generally similar in appearance to arbors at the first viewing (i.e., they appeared healthy, with no apparent pathological changes). Finally, there was usually net growth of the dendritic arborizations over time; chronically damaged cells would be more likely to show retraction.

All told, it seems unlikely that damage to neurons can account for the long-term changes we describe. On the other hand, neuronal damage as a result of our procedure might have affected the kinetics of these changes (particularly at early times) or the balance between extension and retraction.

\section{Possible bases for ongoing dendritic change of superior cervical ganglion cells}

Although the animals that we observed were adult by the criterion of reproductive competence (achieved in the mouse at about 8 weeks postnatal), mice of this age are still growing. Thus, the average final weight of the animals studied increased $9 \%$ over the starting weight after 2 weeks, $14 \%$ after a month, $30 \%$ after 2 months, and $31 \%$ after 3 months.

Several observations in the autonomic nervous system of mammals suggest that the geometry of ganglion cells is related to animal size (Purves and Lichtmann, 1985b; Purves et al., 1986). For instance, the dendritic arbors of superior cervical ganglion cells in different mammalian species increase systematically in length, complexity, and number of primary dendrites as a function of increasing species size (Purves and Lichtman, 1985b). Similarly, within the developing superior cervical ganglion of the rat, dendritic complexity increases substantially in adult animals (J. Voyvodic, unpublished observations). Since the size of the mice in our study increased appreciably over the intervals studied, a possible stimulus for the ongoing change of the dendritic arbors of these cells may be the continued growth of their targets (Purves et al., 1986). Clearly, it would be of considerable interest to carry out similar studies in older animals to determine whether these dendritic changes cease when growth ceases or persist in middle and old age.

Whatever their relation to animal growth may be, the most general description of these changes over time would be that, on average, the dendritic arbors of the ganglion cells become more complex. This conclusion is consistent with developmental studies of Golgi-impregnated neurons in the mammalian CNS (see, for example, Altman, 1972; Sadler and Berry, 1984). However, the dendritic changes that we have described do not reflect a simple extension of the terminals of each process. On the contrary, although the net change was an overall increase in dendritic length and complexity (Figs. 12, 13), both extension and retraction of individual branches were common (Fig. 14; see also Figs. 6 and 8-11).

\section{Implications of dendritic change for synaptic connectivity}

In the superior cervical ganglion of the mouse, as in other mammals, the majority of preganglionic synapses are made on dendritic arbors. Forehand (1985) has estimated that of the 288 synapses made on these ganglion cells on average, about 24 are on the cell body and 264 on the dendritic arbor. Therefore, the changes we have described in the dendritic arbors of these neurons are likely to involve changes in synaptic contacts on the dendritic arbors. Obviously, a dendritic branch that disappears must lose the synaptic contacts that it supported; conversely, a dendritic branch that extends or forms de novo presumably acquires synapses.

The way in which experience engenders permanent changes in the nervous systems of mammals and other higher vertebrates is one of the major puzzles of modern ncurobiology. In general, thinking on this issue has been divided. One view is that such changes are likely to be generated by regulation of the efficacy of anatomically stable synaptic connections. An alternative view is that the long-term effects of experience are encoded in changes in the numbers and locations of connections between nerve cells. The results we have described here provide direct evidence for dynamic changes in young adult nerve cells. The degree to which a dynamic equilibrium of synaptic connectivity might provide a cellular substrate for the long-term storage of information in the mammalian nervous system is clearly an issue that merits further investigation.

\section{References}

Altman, J. (1972) Postnatal development of the cerebellar cortex in the rat. III. Maturation of the components of the granular layer. J. Comp. Neurol. 145: 465-514.

Anzil, A. P., A. Bieser, and A. Wernig (1984) Light and electron microscopic identification of nerve terminal sprouting and retraction in normal adult frog muscle. J. Physiol. (Lond.) 350: 393-399.

Forehand, C. J. (1985) Density of somatic innervation on mammalian autonomic ganglion cells is inversely related to dendritic complexity and preganglionic convergence. J. Neurosci. 5: 3403-3408.

Hadley, R. D., and D. Purves (1985) Ongoing changes in the dendritic arbors of individual superior cervical ganglion neurons visualized in living mice. Soc. Neurosci. Abstr. 11:963.

Haimann, C., A. Mallart, J. Tomas i Ferre, and N.F. Zilber-Gachelin (1981) Patterns of motor innervation in the pectoral muscle of adult Xenopus laevis: Evidence for possible synaptic remodelling. J. Physiol. (Lond.) 310: 241-256.

Kachar, B. (1985) Asymmetric illumination contrast: A method of image formation for video light microscopy. Science 227: 766-768.

Kater, S. D., and R. D. Hadley (1982a) Intracellular staining combined with videofluorescence microscopy for viewing living identified neurons. In Cytochemical Methods in Neuroanatomy, S. Palay and V. Chan-Palay, eds., pp. 441-459, Alan R. Liss, New York.

Kater, S. D., and R. D. Hadley (1982b) Video monitoring of neuronal plasticity. TINS 5: 8-82.

McPheeters, M., and L. M. Okun (1980) Identification and isolation in vitro of presumptive motoneurons marked by retrograde transport of a new fluorescent tracer. Soc. Neurosci. Abstr. 6: 733.

Miller, J. P., and A. I. Selverston (1979) Rapid killing of single neurons by irradiation of intracellularly injected dye. Science 206: 702-704.

Okun, L. M. (1981) Identification and isolation in vitro of neurons marked in situ by retrograde transport. In New Approaches in Developmental Neurobiology (Short Course Syllabus), Society for Neuroscience, San Diego, CA.

Purves, D., and R. D. Hadley (1985) Changes in the dendritic branching of adult mammalian neurones. Nature 315: 404-406.

Purves, D., and J. W. Lichtman (1980) Elimination of synapses in the developing nervous system. Science 210: 153-157.

Purves, D., and J. W. Lichtman (1985a) Principles of Neural Development, Sinauer, Sunderland, MA.

Purves, D., and J. W. Lichtman (1985b) Geometrical differences among homologous neurons in mammals. Science 228: 298-302.

Purves, D., E. Rubin, W. D. Snider, and J. Lichtman (1986) Relation of animal size to convergence, divergence, and neuronal number in peripheral sympathetic pathways. J. Neurosci. 6: 158-163.

Sadler, M., and M. Berry (1984) Remodelling during development of the Purkinje cell dendritic tree in the mouse. Proc. R. Soc. London [Biol.] 221: 349-368.

Sotelo, C., and S. L. Palay (1971) Altered axons and axon terminals in the lateral vestibular nucleus of the rat. Possible example of axonal remodeling. Lab. Invest. 25: 653-671.

Townes-Anderson, E., and G. Raviola (1978) Degeneration and regeneration of autonomic nerve endings in the anterior part of the rhesus monkey ciliary muscle. J. Neurocytol. 7: 583-600.

Van Essen, D. C. (1982) Neuromuscular synapse elimination. In $\mathrm{Neu}$ ronal Development, N. C. Spitzer, ed., pp. 333-376, Plenum, New York.

Wernig, A., J. J. Carmody, A. P. Anzil, E. Hansert, M. Marciniak, and H. Zucker (1984) Persistence of nerve sprouting with features of synapse remodelling in soleus muscles of adult mice. Neuroscience 11: 241-253.

Wernig, A., M. Pecót-Dechavassine, and H. Stöver (1980) Sprouting and regression of the nerve at the frog neuromuscular junction in normal conditions and after prolonged paralysis with curare. J. Neurocytol. 9: 277-303. 